\title{
Neoantigen polypeptide vaccines induce effective antitumor response in colorectal cancer
}

\author{
Yaojun Yu \\ Wenzhou Medical University Second Affiliated Hospital \\ jing zhang ( $\sim$ ZJ839150284@163.com ) \\ Leyi Ni \\ Wenzhou Medical University Second Affiliated Hospital \\ Yuesheng Zhu \\ Wenzhou Medical University Second Affiliated Hospital \\ Hejie Yu \\ Wenzhou Medical University Second Affiliated Hospital \\ Yangyang Teng \\ Wenzhou Medical University Second Affiliated Hospital \\ Limiao Lin \\ Wenzhou Medical University Second Affiliated Hospital \\ Zhanxiong Xue \\ Wenzhou Medical University Second Affiliated Hospital \\ Xiangyang Xue \\ Wenzhou Medical University \\ Xian Shen \\ Wenzhou Medical University Second Affiliated Hospital \\ Haiping Song \\ Affiliated Hospital of Medical College Qingdao University \\ Xiaoping Su \\ Wenzhou Medical University \\ Weihong Sun \\ Affiliated Hospital of Medical College Qingdao University \\ Zhenzhai Cai \\ Wenzhou Medical University Second Affiliated Hospital
}

Wenzhou Medical University Second Affiliated Hospital https://orcid.org/0000-0001-9899-4549

\section{Research}

Keywords: Colorectal cancer; Immunotherapy; Neoantigens; Tumor; Cancer vaccine

Posted Date: April 10th, 2020

DOI: https://doi.org/10.21203/rs.3.rs-22032/v1

License: (1) This work is licensed under a Creative Commons Attribution 4.0 International License. Read Full License 


\section{Abstract}

Background: The role of neoantigens in cancer immunotherapy is crucial. However, the effectiveness and safety of personalized neoantigen vaccines in colorectal cancer (CRC), especially in Chinese population, has not been well studied. This paper mainly explores the feasibility and effectiveness of personalized neoantigen vaccines in CRC treatment.

Methods: Whole-exome sequencing and transcriptome sequencing were used to identify somatic mutations, RNA expression and human leukocyte antigen (HLA) alleles. Neoantigens were predicted, and the immunogenicity of neoantigen candidates was evaluated by ELISPOT in vitro. To verify the immunogenicity in vivo, neoantigen candidates from HLA-A0201 ${ }^{+} \mathrm{PW} 11$ were used to immunized female 68-week-old HLA-A2.1/ $\mathrm{K}^{\mathrm{b}}$-transgenic $(\mathrm{Tg})$ mice. Neoantigen-reactive T cells (NRTs) were induced by immunogenic peptides from autologous HLA-A2.1/ $\mathrm{K}^{\mathrm{b}}$ to adoptive transfer transgenic mice, and C57BL/ $6^{\mathrm{nu} / \mathrm{nu}}$ mice were used for in vivo antitumor response assays.

Results: Compared to medium alone (no peptide) or the unrelated peptide VSV-NP ${ }_{43-69}$, the neoantigens TSHZ3-L523P, RARA-R83H, TP53R248W, EYA2-V333I and NRAS-G12D from Patient 4 (PW4); HAVCR2-F39V, SEC11A-R11L, TASP1-P161L, RAP1GAP-S215R, M0SPD1-V63I and NAV2-D1973N from Patient 10 (PW10); and SMPDL3B-T452M, LRFN3-R118Q and ULK1-S248L from Patient 11 (PW11) induced notable peptide-specific $T$ cell responses. The results indicated that about half of the predicted neoantigens for all 3 patients can stimulate T cell responses and antitumor effects in CRC. In addition, predicted neoantigens from PW11 (HLA-A0201) showed promising antitumor efficacy in HLA-A2.1/ $\mathrm{K}^{\mathrm{b}}$ - $\mathrm{Tg}$ mice and tumor-bearing mouse models.

Conclusion: With the application of next-generation sequencing (NGS) sequencing of patient specimens, neoantigen prediction and a rapid immunoassay system, an evaluation system utilizing in vitro studies and in vivo transgenic and tumor-bearing mouse models can be used to screen strong immunogenic neoantigens in CRC patients. Accurate identification of neoantigens with strong immunogenicity would promote personalized cancer vaccine development.

\section{Introduction}

Colorectal cancer (CRC) is the third most common cause of cancer-related death worldwide(1). At present, the incidence of CRC ranks fifth among systemic malignant tumors in China(2). Surgery is the main treatment strategy for CRC patients, but adjuvant chemotherapy is available only for patients with stage II and most patients with stage III CRC and stage II or III rectal cancer $(3,4)$. Survival has increased over the past 30 years with the introduction of screening programmes and development of new targeted drugs. However, the 5-year relative survival rate of CRC is still only $68 \%$. It is worth noting that approximately one-third of patients undergoing curative surgery for CRC develop recurrent disease because of incomplete tumor resection, and the standard therapies of surgery and adjuvant chemotherapy are often limited by side effects and resistance to chemotherapy $(5,6)$. Therefore, it is highly important to develop other strategies for CRC treatment.

In recent years, immunotherapy for cancer treatment has made great progress $(7,8)$. However, only $<5 \%$ of patients with advanced CRC benefited from programmed cell death protein 1 (PD-1) checkpoint blocking immunotherapy and the efficacy in CRC was limited(9). Personalized neoantigen vaccines, as a form of cancer immunotherapy, have enormous potential to induce potent and long-lasting antitumor effects. With the advent and availability of next-generation sequencing (NGS), the identification of patient-specific neoantigens for personalized vaccines has become feasible. Neoantigen vaccines were shown to be immunogenic and safe with promising clinical effects in several clinical trials of melanoma, glioblastoma and other types of cancer(10, 11). In CRC, HLA-C*08:02-restricted tumorinfiltrating lymphocytes (TIL) immunotherapy targeting oncogene mutation KRAS G12D has been shown to promote tumor regression in one patient(12). However, the effectiveness and safety of personalized neoantigen vaccines in CRC, especially in Chinese population, has not been well studied, so we developed an effective personalized vaccination programme for CRC patients to achieve a significant increase in antitumor effects.

In this research, 13 CRC patients were recruited to study the feasibility and effectiveness of neoantigen polypeptide vaccines. Tumor tissue samples and peripheral blood cells were collected for neoantigens identification. Antitumor effects of personalized neoantigen vaccines for CRC patients were verified by an neoantigen-reactive T cell (NRT)-induced in vitro cytotoxicity assay and in vivo experiments in tumor-bearing mice model. The results indicated that about half of the predicted neoantigens for 3 patients can stimulate T cell responses and antitumor effects in CRC. This study shows that neoantigens can be used to create an ideal cancer vaccine for CRC, thus establishing a path for personalized immunotherapy for CRC patients.

\section{Materials And Methods}




\section{Patient material and cell lines}

Patient tumor samples were obtained from biopsy specimens. A portion of each sample was subjected to formalin fixation and paraffin embedding, and the remaining tumor tissue was carefully manually dissociated in collagenase D (200 U ml-1) and DNase I (20 U ml-1) (Roche Life Sciences). The dissociated samples were suspended in the solution for a period of time, transferred to a tightly sealed plastic bag, incubated together, and periodically stirred for 30-60 minutes with a laboratory stirrer.

The study population included adult patients with a histologically or cytologically confirmed diagnosis of CRC from the Second Affiliated Hospital of Wenzhou Medical University between February 2017 and February 2019. All donors gave written informed consent for the collection of tissue specimens. This study involving human samples was approved by the Medical Ethics Committee of the Second Affiliated Hospital, Wenzhou Medical University (MEC numbers: LCKY2018-67), in line with the Helsinki Declaration, and always adhered to the privacy rights of human subjects. The procedure was performed in strict accordance with the protocol.

Cell lines were obtained from American Type Culture Collection (ATCC, Manassas, VA, USA). Peripheral blood mononuclear cells (PBMCs) were the original material harvested for immunological monitoring or sample manufacturing; these cells were isolated by density gradient centrifugation using Ficoll-Hypaque (Amersham Biosciences) and collected from the buffy coat of peripheral blood samples from healthy donors or CRC patients, thereby producing immature dendritic cells (DCs). H522 cells were cultured under standard conditions described by the ATCC, and the cells were re-certified by short tandem repeat (STR) analysis at ATCC, while a cell bank for mycoplasma was generated.

\section{Next-generation Sequencing (ngs)}

For RNA sequencing (RNA-seq), RNeasy Mini Kit was utilized to extract RNA from fresh frozen tumor samples. The construction of RNAseq libraries were performed using the TruSeq Stranded mRNA Library Prep kit (Illumina) (for cell suspensions). All libraries were sequenced paired-end 50 nt on an Illumina HiSeq 2500 sequencing platform by using two Illumina-based TruSeq SBS Kits v3-HS 50 cycles. Paired ends (100 nt) were sequenced using TruSeq SBS Kit v3-HS (Illumina) (200-cycles) to distribute the MZ-GaBa-018 cell line and its matched PBMC genome-wide sequencing library in 4 lanes.

For WES sequencing, DNA was extracted from the fresh-frozen tumor samples or cultured tumor cells using DNeasy Blood and Tissue Kit, purchased from Qiagen. Genomic DNA was cut, end-repaired, simultaneously connected to the bar-coded Illumina sequencing adapters, amplified, and size-selected. Whole-exome capture was performed using Agilent Sure Select Human All Exon 44-Mb version 2.0 bait set (Agilent Technologies), The resulting libraries were quantified by qPCR, and 76-base paired-end reads were pooled and sequenced using HiSeq 2000 or 2500 sequencers (Illumina).

\section{Bioinformatics And Mutation Discovery}

All mutations in a single patient were analysed using the Python programming language. DNA libraries required at least $150 \times 10^{6}$ pairedend $50 \mathrm{nt}$ reads, while RNA libraries required at least $75 \times 10^{6}$ paired-end $50 \mathrm{nt}$ reads. For mutation detection, DNA reads were consistent with the reference genomes hg19. Duplicate exomes from tumor and matched normal samples were analysed for single-nucleotide variants. Identification and screening of sites assumed to be homozygous genotypes in normal samples, thus preserving a high degree of trust in single-nucleotide variation. Further examination of the remaining sites was performed to determine the presumed homozygous or heterozygous mutation event. Screening suspicious sites to exclude potential false positives, and testing the sum of duplicates and duplicates respectively, and merging duplicates. We compared the genomic coordinates of the identified variants with the detailed known gene transcription coordinates in the UCSC, and further determined the relationship between the mutations and genes, transcription, expression values derived from RNA-seq, and the changes of potential amino acid sequences.

\section{Hla Typing}

The HLA alleles of each patient were inferred from the WES data using OptiType(13) with the default settings after the reads were filtered by aligning to the HLA region using RazerS version 3.4.0(14).

\section{Neoantigen Candidates Identification}


Non-synonymous somatic mutations, including SNVs, insertion, deletion and frame shift, were used to predict neoantigens by MuPeXI(15), which is a pipeline for neoantigen identification. First, 8-11-amino-acid long mutant peptides were generated for MHC Irestricted neoantigen prediction, and 12-15-amino-acid long mutant peptides were generated for MHC II-restricted neoantigen prediction. Then HLA binding affinity for each peptide was calculated by NetMHCpan4.0(16) and NetMHCllpan3.2(17) for MHC I and MHC II molecules respectively. Expression level of mutant genes was derived from RNA-seq data in transcripts per million (TPM). The number of mismatches between the mutant and normal peptides was considered as the similarity to self-peptides. Mutant allele frequency was detected by the variant caller MuTect2(18). Each peptide was given a priority score based on HLA binding affinity, expression level, similarity to self-peptides and mutant allele frequency. Peptides with priority score larger than 0 were selected as neoantigen candidates.

\section{Synthesis Of Long Peptidesand Final Vaccine Preparation}

The peptides with purity greater than $95 \%$ were analysed by mass spectrometry and were synthesized by GL Biochem (Shanghai, China) using fluorenylmethoxycarbonyl chemistry and purified by reversed-phase high-performance liquid chromatography. The lyophilized peptides were dissolved in dimethyl sulfoxide and diluted to a concentration of $10 \mathrm{mM}$ in phosphate buffer brine (pH 7.4), and continued to be stored as isocyanates at $-80^{\circ} \mathrm{C}$.

\section{Minigenes (tmgs)}

The construction process for tandem minigenes (TMGs) has been described previously(19). In each TMG construction, 12 small genes were included. A TMG plasmid was constructed by linearization with the restriction enzyme Nsil, and in vitro RNA transcription was performed by using each of the encoded linearized plasmids as a template with the mMESSAGE mMACHINE T7 Transcription Kit (Thermo Fisher Scientific); 20 mg of RNA was purified for cell transfection. The remaining PBMC cells after cryopreservation of removal of DC/T cells in this section were thawed and cultured, and minigene RNA was introduced into the PBMCs using a nuclear transfection kit (Amaxa Cell Line Nucleofector Kit V, Lonza). After 24 hours of transfection, these cells were used as stimulatory cells, and the above procedure was performed strictly in accordance with the manufacturer's instructions.

\section{Induction of neoantigen-reactive T cells by coculture with CRC patient-derived peripheral blood lymphocytes (PBLs) in vitro}

Autologous PBMCs from a patient were used to assess the in vitro immunogenicity of candidate neoantigens. We used a simple and rapid culture method to detect and monitor circulating antigen peptide-specific cytotoxic T lymphocyte precursors (CTL-P), which was only slightly modified in previous reports $(20,21)$. Peripheral blood was obtained from patients that had been undergoing postoperative chemoradiotherapy for more than 1 month. PBMCs were isolated and cultured. The human PBMCs were suspended in complete medium (RPMI 1640 medium containing 10\% foetal bovine serum) for collection. PBMC-derived DCs were harvested on the sixth day after cultivation, adjusted for cell concentration using a complete medium for human DCs (RPMI 1640 complete medium supplemented with $500 \mathrm{U} / \mathrm{ml}$ rhGM-CSF and $10 \mathrm{ng} / \mathrm{ml}$ th-4), Frozen T cells collected in RPMI 1640 medium were suspended in 10\% foetal bovine serum and co-culture for 3 stimulations. The cells were collected 7 days after the last stimulation, and positive selection with CD3 immunomagnetic beads (Miltenyi Biotec) was performed. The T cells were selected in strict accordance with the instructions provided by the manufacturer. In vitro gene transcription using the mMESSAGE mMACHINE Ultra Kit (Thermo Fisher) kit was performed. Minigene RNA was transferred into PBMCs by a nuclear transfection kit (Amaxa Cell Line Nucleofector Kit V, Lonza). On day 10, the specific response of T cells to each peptide was assessed by ELISPOT assays. Comparing the response induced by the peptide to that induced by medium alone (no peptide) or the unrelated peptide VSV-NP $43-69$ (STKVALNDLRAYVYQGIKSGNPSILHI), and phytohemagglutinin was used as the positive control. T cell reactivity was assessed by DCs peptide pulses co-cultured with T cells in some cases. Finally, T cell activity was detected by an IFN-y ELISPOT, an ELISA kit and intracellular flow cytometry staining.

\section{Generation Of Neoantigen-specific T Cells In Hla-a2.1/k-transgenic Mice}

All experimental procedures and animal protocols were approved by the AAALAC-accredited Animal Studies Committee of Wenzhou Medical University (AEC numbers:WYDW2019-0924) and were in compliance with all relevant ethical regulations. All procedures are designed in accordance with the ethical standards of the national research council to minimize the suffering and number of animals. All animals used in this study were purchased from the Jackson Laboratory (Bar Harbor, ME, USA). Environmental conditions were a 
temperature of $20 \pm 2{ }^{\circ} \mathrm{C}$, the humidity of $55 \% \pm 10 \%$, the lighting of 350 lux (at bench level) and a $12 / 12 \mathrm{~h} \mathrm{light/dark}$ cycle and All the mice were fed the standard rodent diet with free access to water. The health of the animals was monitored twice daily during the feeding period. No adverse events were observed. All parts of the report follow the ARRIVE Guidelines for animal studies(22).

For the in vivo efficacy evaluation studies, we used eight 6-8-week-old female HLA-A2.1/ $\mathrm{K}^{\mathrm{b}}-\mathrm{Tg}$ mice (mean body weight: $20 \pm 2 \mathrm{~g}$ ), which were raised under standard conditions and cared for in accordance with the animal care facility guidelines(23). Tumor neoantigen peptides from patients with HLA-A0201 were selected and synthesized to vaccinate HLA-A2.1/ $\mathrm{K}^{\mathrm{b}}$ transgenic mice subcutaneously at 0,7 days. $50 \mathrm{mg}$ poly (I: C) was mixed with $100 \mathrm{mg}$ polypeptide each time. Seven days after the last immunization, the spleen of mice was extracted by aseptic operation, and the residual blood was rinsed with sterile saline, soaked in RPMI1640 medium, and the single-cell suspension was obtained by gently grinding the needle core of aseptic syringe on 400 mesh steel net, and filtered through steel net to remove large tissue blocks. After centrifuging $1000 \times \mathrm{g}$ of the filtered single-cell suspension for 5 minutes, the supernatant of the culture medium was discarded and suspended in the low permeable $\mathrm{NH}_{4} \mathrm{Cl}$ solution $\left(0.15 \mathrm{M} \mathrm{NH}_{4} \mathrm{Cl}, 1 \mathrm{M} \mathrm{KHCO}_{3}, 0.1 \mathrm{mM} \mathrm{Na}_{2} \mathrm{EDTA}\right.$, pH 7.2) for 3 minutes to destroy the red blood cells, and then centrifuged and washed twice with RPMI1640 culture medium. The spleen cells of the mice without red blood cells were suspended in RPMI1640 medium containing 10\% fetal bovine serum, counted and placed in 6-well plates. Mouse spleen cells were cultured in vitro and stimulated again with corresponding peptide $(20 \mu \mathrm{M})$. After 7 days, the effector cells were collected and analysed by ELISPOT, intracellular staining and specific killing activity in tumor-bearing mice.

\section{Elispot Assay}

An ELISPOT assay (R\&D Systems, Minneapolis, MN) was performed using a commercial kit. T cells sorted by CD3 immunomagnetic beads were suspended in RPMI 1640 medium supplemented with 10\% foetal bovine serum and used as reacting cells, and the cell concentration was adjusted to $5 \times 10^{6} / \mathrm{ml}$, and the cell suspension was directly transferred to an ELISPOT assay plate $(100 \mu \mathrm{l} /$ well) coated with an anti-IFN-y antibody, PBMC (mutant type (Mut) minigenes), PBMC (wild-type (WT) minigenes) 15 Gy irradiation was used as a stimulating cell and was added to the detection well (100 ul/well). IFN-y-secreting T cell colony was detected by the method described in the IFN-y ELISPOT assay kit instructions. The frequency of nocturnal peptide (80) activation in cytokine-secreting cells was determined by an IFN-y ELISPOT kit (Dakewei). In this research, multiple culture protocols were used to analyse T cell-mediated immune responses. Peptide-stimulated PBMCs were added to two wells for approximately 18 hours or DCs pulsed with a peptide were added and cocultured with the $T$ cells. The plate was washed first, and then a diluted test antibody (1:100 dilution) was added and incubated at $37^{\circ} \mathrm{C}$ for 1 hour. After washing the plate, streptavidin-HRP diluted to the above mentioned year-on-year dilution was added and incubated at the same temperature for 1 hour, and a 3-amino-9-ethylcarbazole (AEC) solution was prepared according to the manufacturers specifications, and added per well. The plate was allowed to incubate at room temperature for approximately 15-20 minutes in darkness, after which time development was stopped by the addition of deionized water. The plates were scanned with an ELISPOT CTL Reader (Cellular Technology Inc) and analysed by ElisPot software (AID), and spots two-times larger than those in the peptide-free (culture medium) control were considered to be $T$ cell reactive positive.

\section{Intracellular Detection Of Ifn-y}

The detection method used to evaluate intracellular IFN-y production was performed as described above. Briefly, in RPMI 1640 medium supplemented with $10 \%$ foetal bovine serum, frozen T cells collected previously were suspended and adjusted to a cell concentration of 4 $\times 10^{6} / \mathrm{ml}$, and $0.5 \mathrm{ml}$ was added to each of the above collected peptide-sensitized autologous DCs, and co-cultured at $37^{\circ} \mathrm{C}, 5 \%$ carbon dioxide (T:DC ratio of 10:1), and the same stimulation was provided three times per week. Recombinant IL-2 (rhIL-2; $20 \mathrm{U} / \mathrm{ml}$ ) was added every 3 days during the culture, the medium was replaced with fresh medium every 2-3 days, and the cells were expanded according to need. The cells were collected 7 days after the last stimulation, and CD3 immunomagnetic beads (Miltenyi Biotec) were used for positive selection of T cells. The sorted cells were subjected to intracellular cytokine staining analysis and fixed in $4 \%$ paraformaldehyde until flow-cytometry analysis (FACScan ${ }^{T M}$ or FACSVantage ${ }^{\mathrm{TM}} \mathrm{SE} ; \mathrm{BD}$ Biosciences). Flow cytometry data were analysed by CellQuest software (BD Biosciences).

\section{In Vitro T Cell Cytotoxicity Assay}

The cytotoxic activity of neoantigen-specific tumour-specific cytotoxic T cells (CTLs) can be tested by a Carboxy fluorescein Succinimidyl Ester (CFSE)/propidium iodide-labelling cytotoxicity assay. HLA-A0201+ $\mathrm{T} 2$ cells loaded with a mutant polypeptide and HLA- 
A0201 ${ }^{+}$SW480 cells transfected with the corresponding mutant polypeptide minigene were used as target cells. For CFSE labeling, target cells were labelled for 10 minutes in PBS containing $4 \mathrm{mM} \mathrm{CFSE} \mathrm{(Invitrogen)} \mathrm{at} 37^{\circ} \mathrm{C}$. Labelling was terminated by adding a $10 \mathrm{x}$ volume of PBS and washing thoroughly with PBS. Cells were seeded in 24-well plates, and the CFSE-labelled cells at different effector:target cell ratios (E:T ratios) were incubated with $T$ cells for 6 hours. Then, propidium iodide (Sigma-Aldrich) was added directly to detect cell death rate, and the samples were analysed by flow cytometry.

Cytotoxicity assays were determined by CCK8 assay(24). The formula for determining percent specific lysis was as follows: cell cytotoxicity $=(1-(A e-A b) /(A c-A b)) \times 100 \%$, where $A e$ is the absorbance of the experimental group, Ac is the absorbance of the control group, and $\mathrm{Ab}$ is the absorbance of a blank well.

\section{Adoptive Immunotherapy In Tumor-bearing Nude Mice}

For the adoptive transfer model, we used 6-8-week-old female C57BL/6 ${ }^{\mathrm{nu} / \mathrm{nu}}$ mice (mean body weight: $20 \pm 2 \mathrm{~g}$ ). Splenocytes from vaccinated Tg mice in each group were stimulated with $20 \mu \mathrm{M}$ HAVCR2-F39V, SEC11A-R11L and ULK1-S248L for 7 days. MAD-MB-468hPEBP4 tumor cells $\left(5 \times 10^{6}\right)$ were injected into the C57BL/ $6^{\text {nu/nu }}$ mice breast fat pads, forming $100 \%$ homogeneous tumors. Three days later, the mice were injected intravenously with splenocytes $\left(1 \times 10^{8}\right.$ cells per mouse) from vaccinated HLA-A2.1/K $\mathrm{K}^{\mathrm{b}}$ transgenic mice in each group that were stimulated with $20 \mu \mathrm{M}$ HAVCR2-F39V, SEC11A-R11L and ULK1-S248L for 7 days as described in the section detailing the protocol used for the cytotoxicity test. This adoptive infusion was performed two times 1-week intervals, and intraperitoneal injection of $2000 \mathrm{U}$ of hlL-2 every 2 days. Control mice accepted transgenic mice splenocytes by immunization with non-pulsed DCs or injected with only IL-2. The size of the tumor (two dimensional) was measured three times per week, and the HLA-A2.1/K mice were sacrificed on day 80 after tumor inoculation.

\section{Statistical analysis}

The two-tailed Student's t-test was used to determine statistical significance of the differences between means. Tumor sizes were compared between groups using the Mann-Whitney U-test. Survival analyses were plotted using Kaplan-Meier curves. Values of $P<0.05$ were considered as statistically significant. All statistical tests were performed using SPSS manager software (v21; IBM Inc.)

\section{Results}

\section{Somatic mutation identification and neoantigen prediction for CRC patients}

We recruited 13 patients with CRC (Table 1) and collected tumor tissue and peripheral blood cell samples from each patient for WES and RNA-sEq. The tumor tissue sample of patient PW1 was not qualified for sequencing, so we only got sequencing results for 12 patients. HLA alleles of 12 patients were identified by OptiType using WES data, and the results are listed in Supplementary Table 1. A median of 54 non-synonymous somatic mutations (range 29-89) were identified (Fig. 1A) and selected to predict neoantigens that bind to MHC I and II molecules. A median of $36 \mathrm{MHC}$ 1-restricted neoantigens (range 16-61) were identified by MuPeXI.I (priority score >0), and a median of $15 \mathrm{MHC}-I I$ restricted neoantigens (range 9-28) were identified by MuPeXI.II (priority score >0) (Supplementary Table 2). Among all predicted neoantigens, a median of 9 neoantigens (range 5-18) are strong binders with \%rank less than 0.5 , and a median of 46 neoantigens (range 22-72) are weak binders with \%rank larger than 0.5 (Fig. 1B) 
Table 1

Clinical characteristics of 13 patients receiving personalized immunotherapy

\begin{tabular}{|c|c|c|c|c|c|c|c|c|c|}
\hline $\begin{array}{l}\text { Patient } \\
\text { ID }\end{array}$ & Age & $\operatorname{sex}$ & $\begin{array}{l}\text { Primary } \\
\text { tumor }\end{array}$ & Pathology & Grade & $\begin{array}{l}\text { TNM } \\
\text { (Stage) }\end{array}$ & $\begin{array}{l}\text { Metastatic } \\
\text { Sites }\end{array}$ & Prior Therapy & $\mathrm{PS}^{*}$ \\
\hline PW1 & 78 & $\mathrm{~F}$ & colon & Adenocarcinoma & G3 & $\begin{array}{l}\text { T4bN2bM1b } \\
\text { (IVB) }\end{array}$ & $\begin{array}{l}\text { Liver, } \\
\text { Peritumor and } \\
\text { mesenteric } \\
\text { lymph nodes }\end{array}$ & Surgery & 1 \\
\hline PW2 & 59 & M & colon & Adenocarcinoma & $\mathrm{G} 2$ & $\begin{array}{l}\text { T3N2bM0 } \\
\text { (IIIC) }\end{array}$ & $\begin{array}{l}\text { Peritumor } \\
\text { lymph node }\end{array}$ & $\begin{array}{l}\text { Surgery, Oxaliplatin + } \\
\text { capecitabine }\end{array}$ & 0 \\
\hline PW3 & 33 & $\mathrm{~F}$ & colon & Adenocarcinoma & $\mathrm{G} 2$ & $\begin{array}{l}\text { T3N0M1 } \\
\text { (IV) }\end{array}$ & $\begin{array}{l}\text { Liver, Lung, } \\
\text { ovary, brain, } \\
\text { bone }\end{array}$ & $\begin{array}{l}\text { Oxaliplatin + tegafur, } \\
\text { Bevacizumab,Oxaliplatin + } \\
\text { capecitabine, Surgery, } \\
\text { Irinotecan + 5-Fu + calcium } \\
\text { folinatc, Apixaban, } \\
\text { Oxaliplatin + raltitrexed }\end{array}$ & 4 \\
\hline PW4 & 52 & $\mathrm{~F}$ & rectum & Adenocarcinoma & $\mathrm{G} 2$ & $\begin{array}{l}\text { T3N1aM1 } \\
\text { (IV) }\end{array}$ & $\begin{array}{l}\text { Lung, } \\
\text { Peritumor } \\
\text { lymph node }\end{array}$ & $\begin{array}{l}\text { Surgery, Bevacizumab + } \\
\text { oxaliplatin } \\
\text { +capecitabine }\end{array}$ & 1 \\
\hline PW5 & 72 & $\mathrm{~F}$ & colon & Adenocarcinoma & $\mathrm{G} 2$ & $\begin{array}{l}\text { T3N0M1 } \\
\text { (IV) }\end{array}$ & Liver & $\begin{array}{l}\text { Surgery, Oxaliplatin + } \\
\text { cetuximab } \\
\text { +capecitabine }\end{array}$ & 1 \\
\hline PW6 & 71 & M & colon & Adenocarcinoma & $\mathrm{G} 2$ & $\begin{array}{l}\text { T4bNOM1 } \\
\text { (IV) }\end{array}$ & Liver & Surgery & 1 \\
\hline PW7 & 74 & $M$ & rectum & Adenocarcinoma & $\mathrm{G} 2$ & $\begin{array}{l}\text { T3N1bM1 } \\
\text { (IV) }\end{array}$ & $\begin{array}{l}\text { Liver, } \\
\text { Mesenteric } \\
\text { and tumor } \\
\text { distal lymph } \\
\text { nodes }\end{array}$ & $\begin{array}{l}\text { Surgery, Oxaliplatin + } \\
\text { capecitabine }\end{array}$ & 1 \\
\hline PW8 & 59 & $\mathrm{~F}$ & colon & Adenocarcinoma & G1-2 & $\begin{array}{l}\text { T4aN0M1 } \\
\text { (IV) }\end{array}$ & Liver & $\begin{array}{l}\text { Surgery, Oxaliplatin + } \\
\text { cetuximab } \\
\text { +capecitabine, Irinotecan + } \\
5 \text {-Fu } \\
\text { +calcium folinatc + } \\
\text { cetuximab }\end{array}$ & 1 \\
\hline PW9 & 49 & $\mathrm{~F}$ & rectum & Adenocarcinoma & $\mathrm{G} 2$ & $\begin{array}{l}\text { T3N2M1 } \\
\text { (IV) }\end{array}$ & $\begin{array}{l}\text { Left adnexa, } \\
\text { tumor distal } \\
\text { lymph nodes }\end{array}$ & $\begin{array}{l}\text { Surgery, Oxaliplatin + } \\
\text { capecitabine }\end{array}$ & 0 \\
\hline PW10 & 66 & $\mathrm{~F}$ & rectum & Adenocarcinoma & $\mathrm{G} 2$ & $\begin{array}{l}\text { T4aN1bM1 } \\
\text { (IV) }\end{array}$ & $\begin{array}{l}\text { Uterus, Right } \\
\text { fallopian tube, } \\
\text { Peritumor } \\
\text { lymph nodes }\end{array}$ & $\begin{array}{l}\text { Surgery, Oxaliplatin + } \\
\text { capecitabine }\end{array}$ & 1 \\
\hline PW11 & 57 & M & rectum & Adenocarcinoma & G3 & $\begin{array}{l}\text { T4N2M1 } \\
\text { (IV) }\end{array}$ & $\begin{array}{l}\text { Bladder, } \\
\text { Peritumor and } \\
\text { left internal } \\
\text { iliac artery } \\
\text { lymph nodes, } \\
\text { Napes and } \\
\text { omentum } \\
\text { majus } \\
\text { nodules }\end{array}$ & $\begin{array}{l}\text { Surgery, Oxaliplatin + } \\
\text { capecitabine,Bevacizumab, } \\
\text { Irinotecan + capecitabine, } \\
\text { Bevacizumab + irinotecan } \\
\text { + capecitabine, } \\
\text { Bevacizumab + raltitrexed, } \\
50 \text { Gy/25F radiotherapy }\end{array}$ & 3 \\
\hline PW12 & 89 & $M$ & colon & Adenocarcinoma & $\mathrm{G} 2$ & $\begin{array}{l}\text { T3N1bM1 } \\
\text { (IV) }\end{array}$ & $\begin{array}{l}\text { Liver, Lung, } \\
\text { Mesenteric } \\
\text { lymph nodes }\end{array}$ & Surgery & 2 \\
\hline
\end{tabular}

*PS: Performance status: ECOG, Eastern Cooperative Oncology Group. 


\begin{tabular}{|lllllllll|}
\hline $\begin{array}{l}\text { Patient } \\
\text { ID }\end{array}$ & Age & sex & $\begin{array}{l}\text { Primary } \\
\text { tumor }\end{array}$ & Pathology & Grade $\begin{array}{l}\text { TNM } \\
\text { (Stage) }\end{array}$ & $\begin{array}{l}\text { Metastatic } \\
\text { Sites }\end{array}$ & Prior Therapy \\
\hline PW13 & 68 & M & colon & Adenocarcinoma & G3 & $\begin{array}{l}\text { T4aN2M1 } \\
\text { (IV) }\end{array}$ & $\begin{array}{l}\text { Peritumor, } \\
\text { mesenteric } \\
\text { and }\end{array}$ & $\begin{array}{l}\text { Surgery, Oxaliplatin + } \\
\text { capecitabine,Bevacizumab } \\
\text { +oxaliplatin } \\
\text { +capecitabine, } \\
\text { lymph nodes } \\
\text { Bevacizumab, } \\
\text { Bevaciumab + } \\
\text { capecitabine }\end{array}$ \\
\hline
\end{tabular}

*PS: Performance status: ECOG, Eastern Cooperative Oncology Group.

\section{Validation Of Neoantigen Immunogenicity In Vitro By Elispot}

PBMCs of patient PW4, PW10 and PW11 were used to evaluate the immunogenicity of the neoantigen candidates, because they had sufficient PBMCs. Top 20 neoantigen candidates for patient PW4 and PW11 were augmented to 27-amino-acid long for peptide synthesis, but only 10 peptides for each patient were successfully synthesized. To further validate the immunogenicity of the polypeptides in HLAA2.1/K ${ }^{\mathrm{b}}$-Tg mice, $9 \mathrm{HLA}-\mathrm{A} 0201$-restricted neoantigen candidates from patient PW11 were also successfully synthesized (Supplementary Table 3). A simple and rapid method was used to determine the peptide immunogenicity in cancer patient(25) with a slight modification. The immunogenicity of each synthesized polypeptide was detected in PBMCs from the corresponding patient. ELISPOT analysis showed that compared to medium (no peptide) or the unrelated peptide VSV-NP $43-69$ (STKVALNDLRAYVYQGIKSGNPSILHI), 5 neoantigen candidates from PW4 (TSHZ3-L523P, RARA-R83H, TP53-R248W, EYA2-V333I, NRAS-G12D), 5 neoantigen candidates from PW10 (HAVCR2-F39V, SEC11A-R11L, SMPDL3B-T452M, LRFN3-R118Q, ULK1-S248L), and 4 neoantigen candidates from PW11 (TASP1-P161L, RAP1GAP-S215R, MOSPD1-V63I, NAV2-D1973N) induced obvious peptide-specific T cell responses. In particular, TP53-R248W and NRASG12D from PW4; TASP1-P161L, RAP1GAP-S215R and NAV2-D1973N from PW11; and HAVCR2-F39V, SEC11A-R11L, and ULK1-S248L from PW11 induced the most obvious antigen-specific T cell responses (Fig. 2A-C).

\section{Neoantigen-specific T-cell responses can be induced in vitro in PBLs from CRC patients}

Adoptive T cell therapy has successfully been applied to treat many human solid tumors. There is growing evidence that NRTs can achieve tumor regression in patients receiving adoptive cell therapy $(26,27)$. In addition, to evaluate the ability of NRTs to respond to Mut and WT peptides, we used HLA-A0201 ${ }^{+}$autologous DCs from PW11 loaded with HAVCR2-F39V, SEC11A-R11L, or ULK1-S248L to stimulate PBMCs to generate NRT cells in vitro. Mutated peptide-specific NRTs were produced in vitro and the immune responses were compared with WT peptide-induced NRTs. ELISPOT analysis showed that the NRTs secreted higher levels of IFN-y in response to Mut peptide stimulation than in response to the corresponding WT peptide stimulation (Fig. 3A).

To further examine the ability of NRTs to kill target cells, HLA-A0201+ ${ }^{+} 2$ cells loaded with a mutant polypeptide and HLA-A0201 ${ }^{+}$SW480 cells transfected with the corresponding mutant gene minigene were used as target cells. The results are shown in Fig. 3B-D. At different E:T ratios, bulk NRTs against HAVCR2-F39V, SEC11A-R11L and ULK1-S248L, especially NRT against SEC11A-R11L and ULK1-S248L, can significantly kill target cells expressing the corresponding antigen. No significant killing effect was detected with T2 cells not loaded with peptide or loaded with the irrelevant peptide VSV-NP $43-69$ or with SW480 cells that did not express the mutant peptide.

\section{HLA-A2.1-restricted neoantigens can induce NRT cells in HLA-A2.1/K b transgenic mice in vivo}

To evaluate the immunogenicity of candidate polypeptides in vivo, we selected the mutant polypeptides HAVCR2-F39V, SEC11A-R11L, and ULK1-S248L from HLA-A0201 ${ }^{+}$PW11, which were confirmed to be highly immunogenic in vitro, to immunize $8 \mathrm{Tg}$ mice. On days 0 and 7 , HAVCR2-F39V, SEC11A-R11L and ULK1-S248L (100 $\mu \mathrm{g} /$ per peptide) and $50 \mu \mathrm{g}$ of poly (I:C) were mixed and subcutaneously injected to immunize transgenic mice. Splenocytes were harvested 7 days after the last immunization, and some splenocytes were evaluated by IFNY ELISPOT. The remaining splenocytes were cultured for 7 days under stimulation with the corresponding peptide, and T cells were collected for cytotoxicity analysis. The ELISPOT results showed that the NRTs against SEC11A-R11L and ULK1-S248L from HLA-A2.1/K ${ }^{b}$ 
mice produced a stronger IFN-y-secretion response under stimulation with the corresponding peptide than under stimulation with the WT peptide. Stimulation with medium (no peptide) and the unrelated peptide VSV-NP 43 - 69 produced only a background-level secretion response. However, the NRTs against HAVCR2-F39V produced only background-level secretion responses in response to stimulation with various peptides (Fig. 4A). In a cytotoxicity assay, the NRTs against SEC11A-R11L and ULK1-S248L NRTs significantly killed T2 cells loaded with the corresponding mutant peptide and SW480-minigene expressing the corresponding antigen. No significant killing was detected for T2 cells that were not loaded with the peptide or loaded with the irrelevant peptide VSV-NP $43-69$ or SW480 cells that did not express the mutant peptide (Fig. 4C, 4D). NRTs did not exhibit significant cytotoxicity in response to HAVCR2-F39V (Fig. 4B). No adverse reactions were observed in mice throughout the experiment.

\section{Adoptive NRT immunotherapy of C57BL/6 nu/nu mice bearing human CRC tumors}

To determine whether the SEC11A-R11L and ULK1-S248L peptides can be used as a potent vaccine to limit tumor growth in vivo. We established HLA-A2.1/K $\mathrm{K}^{\mathrm{b}}$-Tg mouse-derived NRTs, which were adoptively transferred into SW480-minigene human CRC-bearing $\mathrm{C} 57 \mathrm{BL} / 6^{\mathrm{nu} / \mathrm{nu}}$ mice. Thus, we developed an adoptive transfer therapy model in nude mice. As shown in Fig. 5A, the mice subjected to adoptive transfer of SEC11A-R11L and ULK1-S248L-stimulated NRTs exhibited significantly delayed tumor growth, whereas CTLs induced by irrelevant peptide could not prevent tumor growth. In addition, in the SEC11A-R11L and ULK1-S248L vaccination groups, 7/10 (70\%) of the ULK1-S248L-vaccinated mice and 6/10 (60\%) of the SEC11A-R11-vaccinated mice showed significant long-term survival after tumor inoculation (over 80 days) (Fig. 5B). All the mice of control group died on days 20 to 40 . Therefore, these results indicate that immunization with the SEC11A-R11L and ULK1-S248L peptides induces an effective antitumor response in vivo. In this study, there were too few mice to determine whether these represented different tissue cell types that respond to neoantigen polypeptide. No adverse reactions were found in the experimental and control mice.

\section{Discussion}

Cancer immunotherapy is rapidly evolving and has been effectively translated into the clinical. Personalized immunotherapy has demonstrated the ability to enhance NRT cell reactivity, which will further enhance the antitumor effect of cancer immunotherapy on malignant tumors(28), but research and application of neoantigen vaccines in CRC, especially in Chinese population, have been rarely reported. To study the effectiveness of neoantigen vaccines on $\mathrm{CRC}$, we established a pipeline to predict and validate neoantigens for CRC patients. We identified neoantigens for $12 \mathrm{CRC}$ patients and validated the immunogenicity of neoantigens for 3 patients. About half of the predicted neoantigens for all 3 patients show immunogenicity to activate T-cell response in patient PBMCs. In addition, predicted neoantigens from PW11 (HLA-A0201) showed promising antitumor efficacy in HLA-A2.1/K ${ }^{b}$ transgenic mice and tumor-bearing mouse models.

In one previous study of T-cell transfer therapy for CRC, Tran et al. screened cultures of TIL from patients for reactivity against all identified mutant neoepitopes and identified one neoepitope KRAS G12D that could stimulate CD8 ${ }^{+}$T-cell response(12). The method of identifying neoantigens was laborious and time-consuming. Thus, in our study, we used computational tools to identify neoantigens from hundreds of mutations. However, one of the most crucial problems of in silico neoantigen identification is the low immunogenicity of predicted neoantigen candidates. State-of-art tools that regard binding affinity between peptide and MHC molecules as the main consideration, such as NetMHCpan and NetMHCIIpan, can only achieve a low validation rate about 20-30\%(29). Therefore, we used MuPeXI which is a computational tool that integrates binding affinity, RNA expression level, mutant allele frequency and similarity to selfpeptidesas factors to determine the probability of neoantigen candidates to activate immune responses(15). According to our results, the validation rate increased to about $50 \%$ by using MuPeXI to predict neoantigens.

Although we and previous studies have demonstrated the immunogenicity and antitumor effects of the neoantigens(10, 11, 30, 31), the neoantigen-based vaccine is less effective against solid tumors, especially for advanced solid tumors. Recently, there has been increasing evidence that adoptive T-cell therapy targeting neoantigens has successfully been applied to treat many human solid tumors(26), further confirming the application of NRTs based personalized tumor therapy. We used HLA-A2.1-restricted neoantigens to induce NRTs in HLAA2.1/ $\mathrm{K}^{\mathrm{b}}$ transgenic mice and transferred the Tg mouse-derived lymphocytes into human CRC tumor-bearing C57BL/6 ${ }^{\text {nu/nu }}$ mice. The CRC mice model show significant tumor regression, which indicates the effectiveness of NRT cell therapy in CRC.

\section{Conclusion}


Our research confirmed the presence of a large number of neoantigens in CRC. Our research demonstrates that these neoantigens can be used as an ideal cancer treatment in CRC.

\section{Abbreviations}

CRC: Colorectal cancer; Tg: Transgenic; PBMCs: Peripheral blood mononuclear cells; NRTs: Neoantigen-reactive T cells; PD-1:

Programmed cell death protein 1; TIL: Tumor-infiltrating lymphocytes; NGS: Next-generation sequencing; HLA: Human leukocyte antigen; DCs: Dendritic cells; STR: Short tandem repeat; RNA-seq: RNA sequencing; TPM: Transcripts per million; TMGs: Tandem minigenes; CTLP: Cytotoxic T lymphocyte precursors; CTLs: Tumour-specific cytotoxic T cells; PBLs: Peripheral blood lymphocytes; CFSE: Carboxyfluorescein succinimidyl ester; WT: Wild-type; Mut: Mutant type; E:T: Effector: target

\section{Declarations}

\section{Acknowledgements}

The authors thank the members of our laboratories for their contributions and helpful discussions. The authors also thank Department of Gastrointestinal Surgery, Second Affiliated Hospital of Wenzhou Medical University (Wenzhou, China) for their generous help.

\section{Authors' contribution}

Yaojun Yu, Jing Zhang and Leyi Ni performed the research and wrote the manuscript. Yuesheng Zhu, Hejie Yu and Yangyang Teng collected clinical samples and corresponding clinical data. Limiao Lin and Zhanxiong Xue made analysis and interpretation of data. Xiangyang Xue, Xian Shen and Haiping Song conducted the in vivo and in vitro work. Xiaoping Su, Weihong Sun and Zhenzhai Cai revised the manuscript. All authors read and approved the final manuscript.

\section{Funding}

The authors disclosed receipt of the following financial support for the research, authorship, and/or publication of this article: This work was supported by the National Natural Science Foundation of China (Grant No. 3197080252); Qingdao Science and Technology Project (for Benefiting the People, 19-6-1-27-nsh); the Wenzhou Major Science and Technology Special Project (2018ZY004) from Wenzhou Science and Technology Bureau; the Public Benefit Technology Research Project of Zhejiang Science and Technology Department (LGF20H160019); and the Research Foundation of Academician Helin Workstation (Item No.19331103).

\section{Availability of data and materials}

The dataset(s) supporting the findings of this study are included within the article.

\section{Ethics approval and consent to participate}

In this study, all investigation and experiments have obtained patients' consent and been approved by the Medical Ethics Committee of the Second Affiliated Hospital, Wenzhou Medical University

\section{Consent for publication}

Written informed consent for publication was obtained from all participants.

\section{Competing interests}

The authors declare that they have no known competing financial interests or personal relationships that could have appeared to influence the work reported in this paper.

\section{References}

1. Siegel RL, Miller KD, Jemal A. Cancer statistics, 2018. CA Cancer J Clin. 2018;68(1):7-30.

2. Chen W, Zheng R, Baade PD, Zhang S, Zeng H, Bray F, et al. Cancer statistics in China, 2015. CA Cancer J Clin. 2016;66(2):115-32.

3. Biondi A, Vacante M, Ambrosino I, Cristaldi E, Pietrapertosa G, Basile F. Role of surgery for colorectal cancer in the elderly. World J Gastrointest Surg. 2016;8(9):606-13. 
4. Biondi A, Grosso G, Mistretta A, Marventano S, Toscano C, Drago F, et al. Laparoscopic vs. open approach for colorectal cancer: evolution over time of minimal invasive surgery. BMC Surg. 2013:S12.

5. Van Loon K, Venook AP. Curable patient with metastatic colorectal cancer: balancing effective therapies and toxicities. J Clin Oncol. 2014;32(10):991-6.

6. Sharif S, O'Connell MJ, Yothers G, Lopa S, Wolmark N. FOLFOX and FLOX regimens for the adjuvant treatment of resected stage II and III colon cancer. Cancer Invest. 2008;26(9):956-63.

7. Pardoll D, Allison J. Cancer immunotherapy: breaking the barriers to harvest the crop. Nat Med. 2004;10(9):887-92.

8. Lesterhuis WJ, Haanen JB, Punt CJ. Cancer immunotherapy-revisited. Nat Rev Drug Discov 2011;10(8):591-600.

9. Llosa NJ, Cruise M, Tam A, Wicks EC, Hechenbleikner EM, Taube JM, et al. The vigorous immune microenvironment of microsatellite instable colon cancer is balanced by multiple counter-inhibitory checkpoints. Cancer Discov. 2015;5(1):43-51.

10. Keskin DB, Anandappa AJ, Sun J, Tirosh I, Mathewson ND, Li S, et al. Neoantigen vaccine generates intratumoral T cell responses in phase Ib glioblastoma trial. Nature. 2019;565(7738):234-9.

11. Ott PA, Hu Z, Keskin DB, Shukla SA, Sun J, Bozym DJ, et al. An immunogenic personal neoantigen vaccine for patients with melanoma. Nature. 2017;547(7662):217-21.

12. Tran E, Robbins PF, Lu YC, Prickett TD, Gartner JJ, Jia L, et al. T-Cell Transfer Therapy Targeting Mutant KRAS in Cancer. N Engl J Med. 2016;375(23):2255-62.

13. Szolek A, Schubert B, Mohr C, Sturm M, Feldhahn M, Kohlbacher O. OptiType: precision HLA typing from next-generation sequencing data. Bioinformatics. 2014;30(23):3310-6.

14. Weese D, Holtgrewe M, Reinert K. RazerS 3: faster, fully sensitive read mapping. Bioinformatics. 2012;28(20):2592-9.

15. Bjerregaard AM, Nielsen M, Hadrup SR, Szallasi Z, Eklund AC. MuPeXI: prediction of neo-epitopes from tumor sequencing data. Cancer Immunol Immunother. 2017;66(9):1123-30.

16. Bugembe DL, Ekii AO, Ndembi N, Serwanga J, Kaleebu P, Pala P. Computational MHC-l epitope predictor identifies $95 \%$ of experimentally mapped HIV-1 clade A and D epitopes in a Ugandan cohort. BMC Infect Dis. 2020;20(1):172.

17. Kardani K, Hashemi A, Bolhassani A. Comparison of HIV-1 Vif and Vpu accessory proteins for delivery of polyepitope constructs harboring Nef, Gp160 and P24 using various cell penetrating peptides. PLoS One. 2019;14(10):e0223844.

18. Kim B, Won D, Jang M, Kim H, Choi JR, Kim TI, et al. Next-generation sequencing with comprehensive bioinformatics analysis facilitates somatic mosaic APC gene mutation detection in patients with familial adenomatous polyposis. BMC Med Genomics. 2019;12(1):103.

19. Tran E, Turcotte S, Gros A, Robbins PF, Lu YC, Dudley ME, et al. Cancer immunotherapy based on mutation-specific CD4+ T cells in a patient with epithelial cancer. Science. 2014;344(6184):641-5.

20. Noguchi M, Sasada T, Itoh K. Personalized peptide vaccination: a new approach for advanced cancer as therapeutic cancer vaccine. Cancer Immunol Immunother. 2013;62(5):919-29.

21. Hida N, Maeda Y, Katagiri K, Takasu H, Harada M, Itoh K. A simple culture protocol to detect peptide-specific cytotoxic T lymphocyte precursors in the circulation. Cancer Immunol Immunother. 2002;51(4):219-28.

22. Kilkenny C, Browne WJ, Cuthill IC, Emerson M, Altman DG. Improving bioscience research reporting: the ARRIVE guidelines for reporting animal research. Osteoarthr Cartil. 2012;20(4):256-60.

23. Slater AM, Cao L. A Protocol for Housing Mice in an Enriched Environment. J Vis Exp. 2015(100):e52874.

24. Liu C, Zheng Y, Tang J, Wang D, Ma Z, Li S, et al. Stimulation of DC-CIK with PADI4 Protein Can Significantly Elevate the Therapeutic Efficiency in Esophageal Cancer. J Immunol Res 2019;2019:6587570.

25. Hida N, Maeda Y, Katagiri K, Takasu H, Harada M, Itoh K. A simple culture protocol to detect peptide-specific cytotoxic T lymphocyte precursors in the circulation. Cancer Immunol Immunother. 2002;51(4):219-28.

26. Chen F, Zou Z, Du J, Su S, Shao J, Meng F, et al. Neoantigen identification strategies enable personalized immunotherapy in refractory solid tumors. J Clin Invest. 2019;129(5):2056-70.

27. Zacharakis N, Chinnasamy H, Black M, Xu H, Lu YC, Zheng Z, et al. Immune recognition of somatic mutations leading to complete durable regression in metastatic breast cancer. Nat Med. 2018;24(6):724-30.

28. Jinushi M, Hodi FS, Dranoff G. Enhancing the clinical activity of granulocyte-macrophage colony-stimulating factor-secreting tumor cell vaccines. Immunol Rev. 2008;222:287-98. 
29. Bjerregaard AM, Nielsen M, Jurtz V, Barra CM, Hadrup SR, Szallasi Z, et al. An Analysis of Natural T Cell Responses to Predicted Tumor Neoepitopes. Front Immunol. 2017;8:1566.

30. Parkhurst MR, Robbins PF, Tran E, Prickett TD, Gartner JJ, Jia L, et al. Unique Neoantigens Arise from Somatic Mutations in Patients with Gastrointestinal Cancers. Cancer Discov. 2019;9(8):1022-35.

31. Tran E, Ahmadzadeh M, Lu YC, Gros A, Turcotte S, Robbins PF, et al. Immunogenicity of somatic mutations in human gastrointestinal cancers. Science. 2015;350(6266):1387-90.

\section{Figures}

A

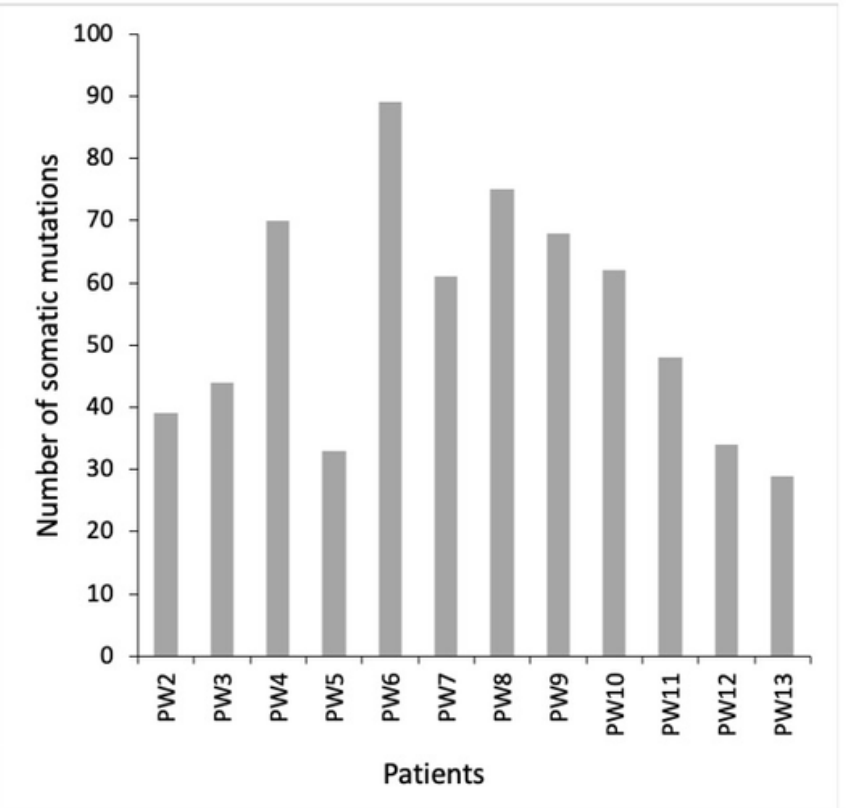

B

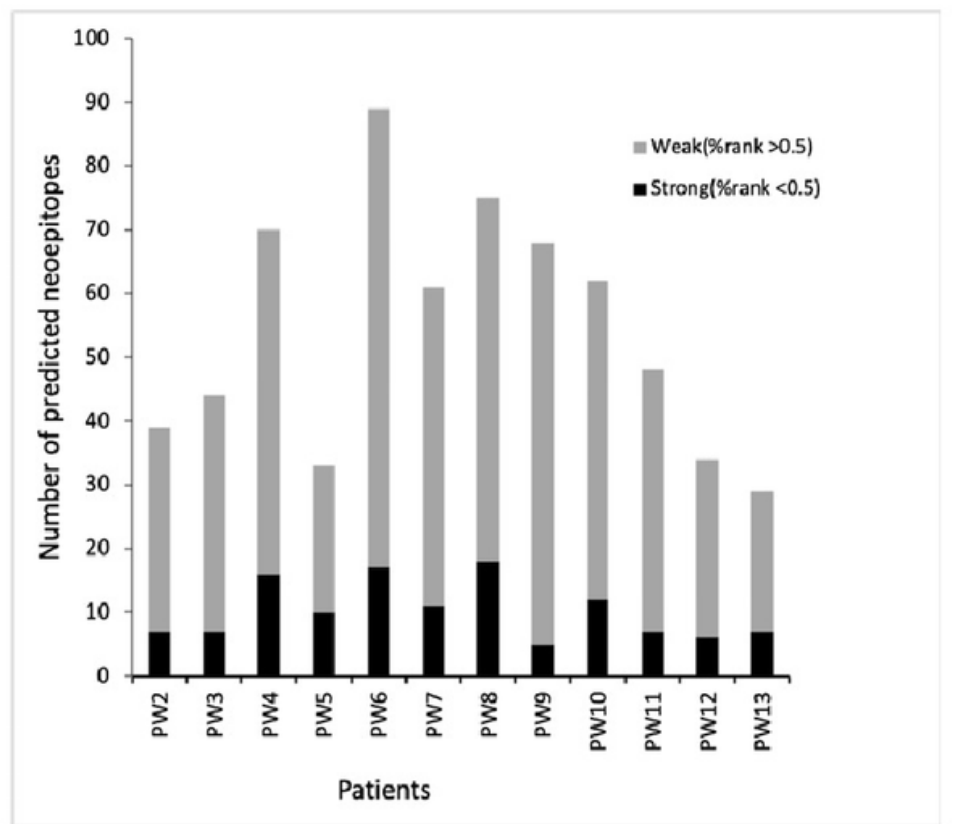

\section{Figure 1}

Frequency of somatic mutations and predicted neoantigens in 12 patients with CRC. (A) WES and RNA-seq were performed in 12 patients with CRC. Tumor-specific non-synonymous somatic mutations were identified. The frequency of somatic mutations of each patient is shown. (B) Neoantigen prediction was performed for each patient. The frequency of neoantigens as well as strong binder $(\%$ rank $<0.5)$ and weak binder $(0.5<\%$ rank $<2)$ of each patient is shown. 

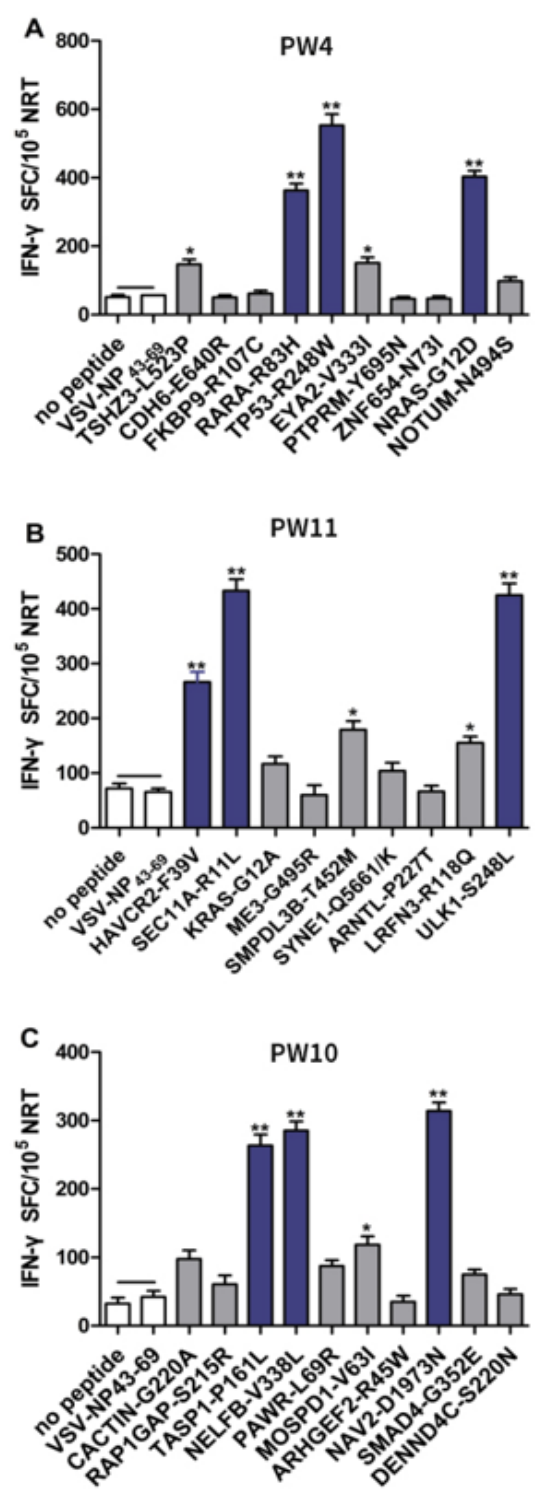

Figure 4

Immunogenicity testing of neoantigens from patients with CRC Autologous PBMCs were stimulated with candidate mutated peptides every 3 days in the presence of IL-2, and on day 10, T-cell responses to each antigen were measured by an IFN-Y ELISPOT assay. The PBMCs in A, B and C are from patients1, 2, and 3 with CRC, respectively. No peptide (medium alone) or VSV-NP43-69 (STKVALNDLRAYVYQGIKSGNPSILHI) stimulation was used as a control. Data are presented as the mean \pm S.D. of three independent experiments. ${ }^{* *} p<0.01$ and $* p<0.05$ compared with IFN-y production by PBMCs stimulated without peptide or with VSV-NP43-69. SFC, spot-forming cell; VSV-NP, vesicular stomatitis virus expressing the influenza nucleoprotein; S.D., standard deviation. 

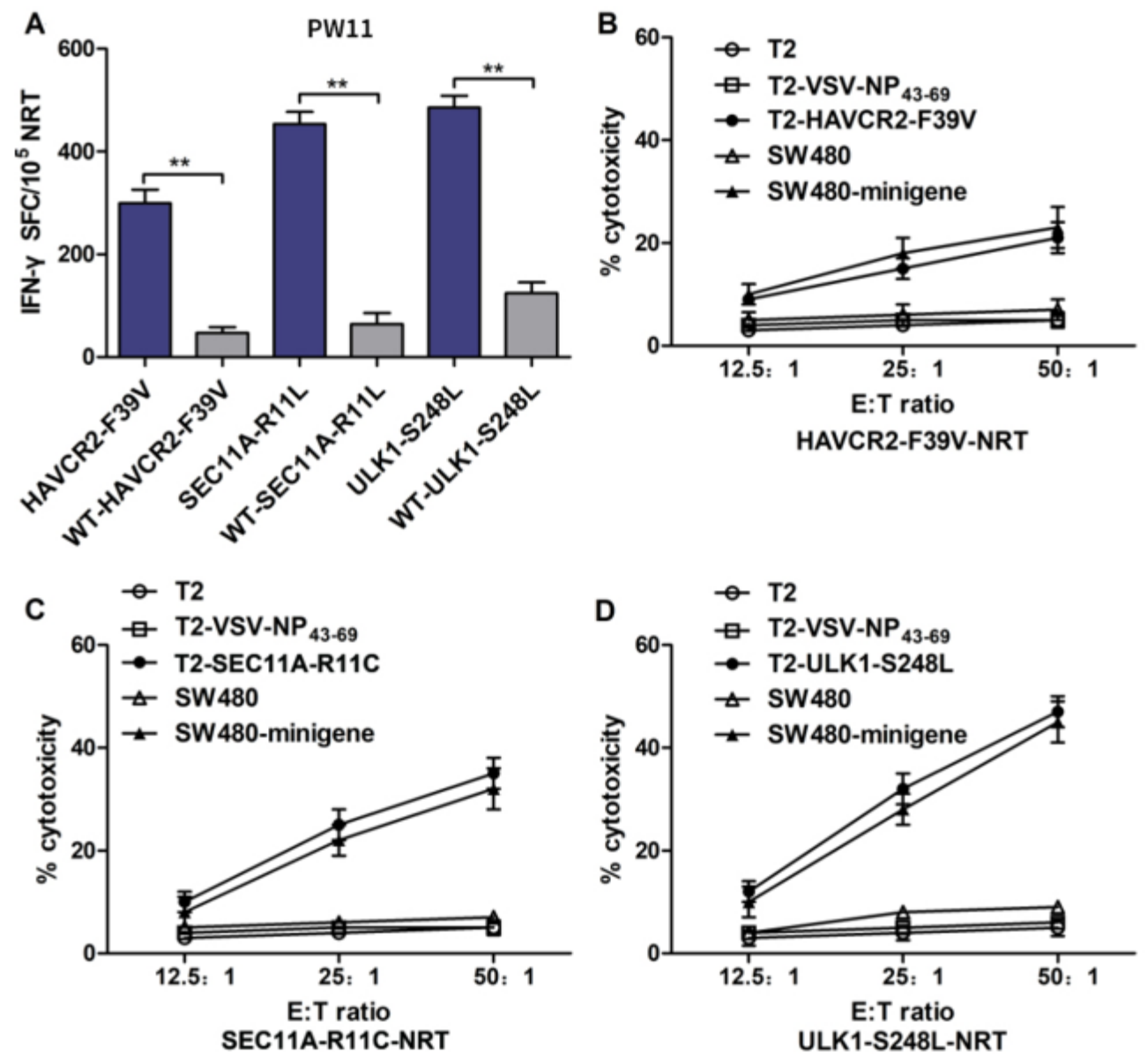

Figure 6

Cytotoxicity of NRTs induced by in vitro stimulation of PBLs from HLA-A0201+ patients with CRC NRTs were induced with autologous HAVCR2-F39V, SEC11A-R11L or ULK1-S248L-pulsed DCs derived from the PBLs of PW11. On day 7 after the third stimulation, the NRTs were harvested for analysis. (A) IFN-y secretion by neoantigen-reactive T-cell lines in response to mutated and wild-type peptides. IFN- $\gamma$ positive SFCs/105 NRTs were detected by cytokine-specific ELISPOT (B, C, and D). The cytotoxicity measured by a CCK8 kit at the specified E:T ratio. Peptide-specific targets were mutated protein-pulsed T2 cells and minimally nucleated SW480 cells (HLA-A2.1+, minigene expression), whereas VSV-NP43-69-pulsed T2 cells, T2 cells alone and SW480 cells alone were used as controls. Data are expressed as the mean $\pm S$.E.M.of three independent experiments. ${ }^{*} \mathrm{p}<0.01$. NRTs, neoantigen-reactive T cells; WT, wild-type; E:T, effector: target; PBL, peripheral blood lymphocyte; SFC, spot-forming cells; S.E.M., standard error of the mean. 

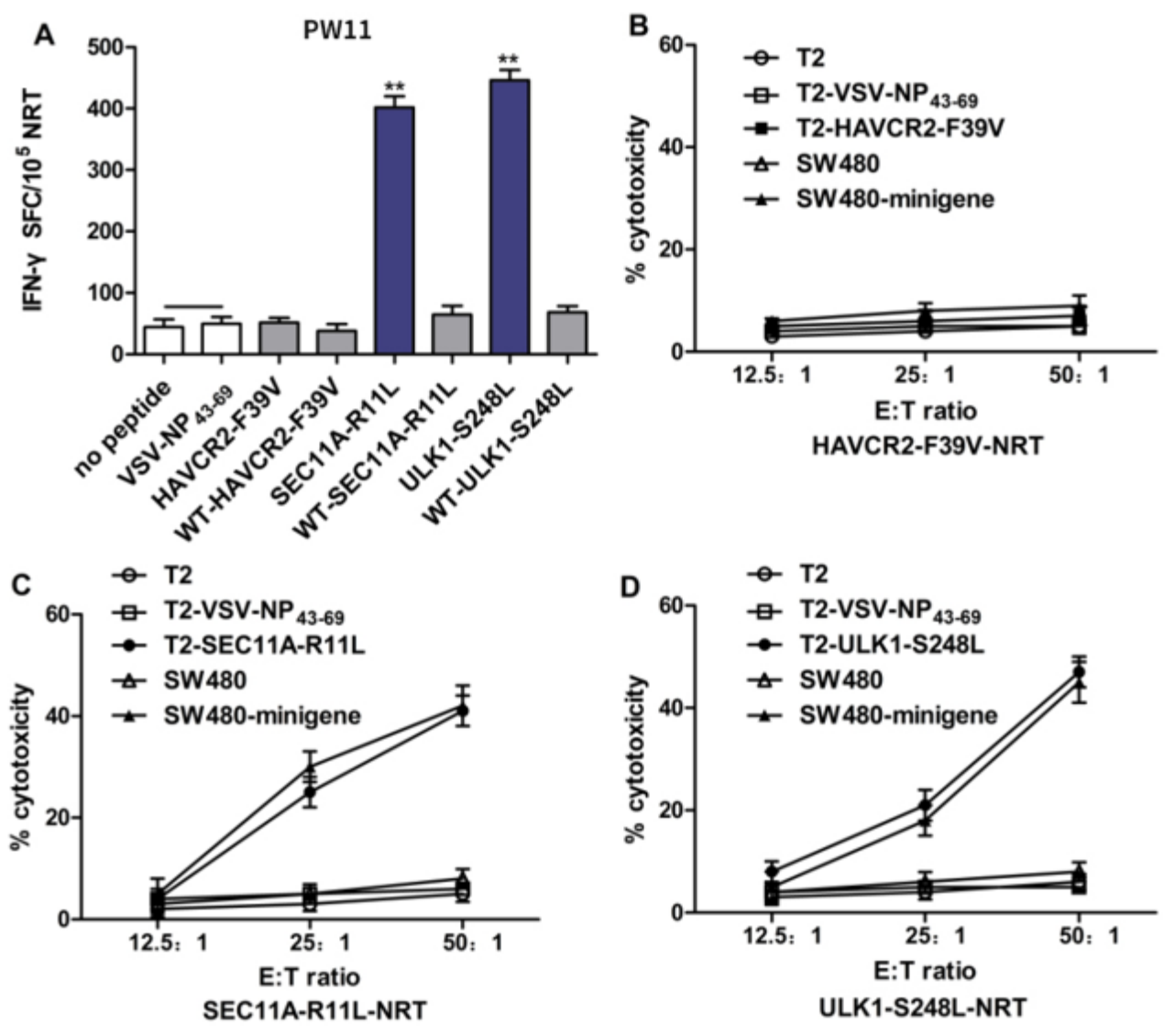

Figure 7

SEC11A-R11L and ULK1-S248L induce more efficient NRT responses than WT epitopes in HLA-A2.1/Kb-Tg mice (A) Splenocytes of mice $(n=5)$ vaccinated with mutated peptides were tested by ELISPOT for recognition of mutated peptides compared to recognition of the corresponding WT sequences. Data are presented as the mean \pm S.D. of three independent experiments. ${ }^{*} \mathrm{p}<0.01$ compared with IFN- $\gamma$ production by splenocytes stimulated without peptide or with VSV-NP43-69 (B, C, and D). Splenocytes from HLA-A2.1/Kb-Tg mice immunized with mutated peptides were restimulated in vitro with the same mutated peptide for 7 days. Ex vivo cytotoxicity against corresponding mutated peptide-pulsed T2 cells and minigene-nucleofected SW480 cells was examined by CCK8 kit assays at the indicated E:T ratio. VSV-NP43-69-pulsed T2 cells, T2 cells alone and SW480 cells alone were used as controls. Data are presented as the mean \pm S.E.M. of three independent experiments. ${ }^{\star \star} \mathrm{p}<0.01$. E:T, effector:target; SFC, spot-forming cell. 


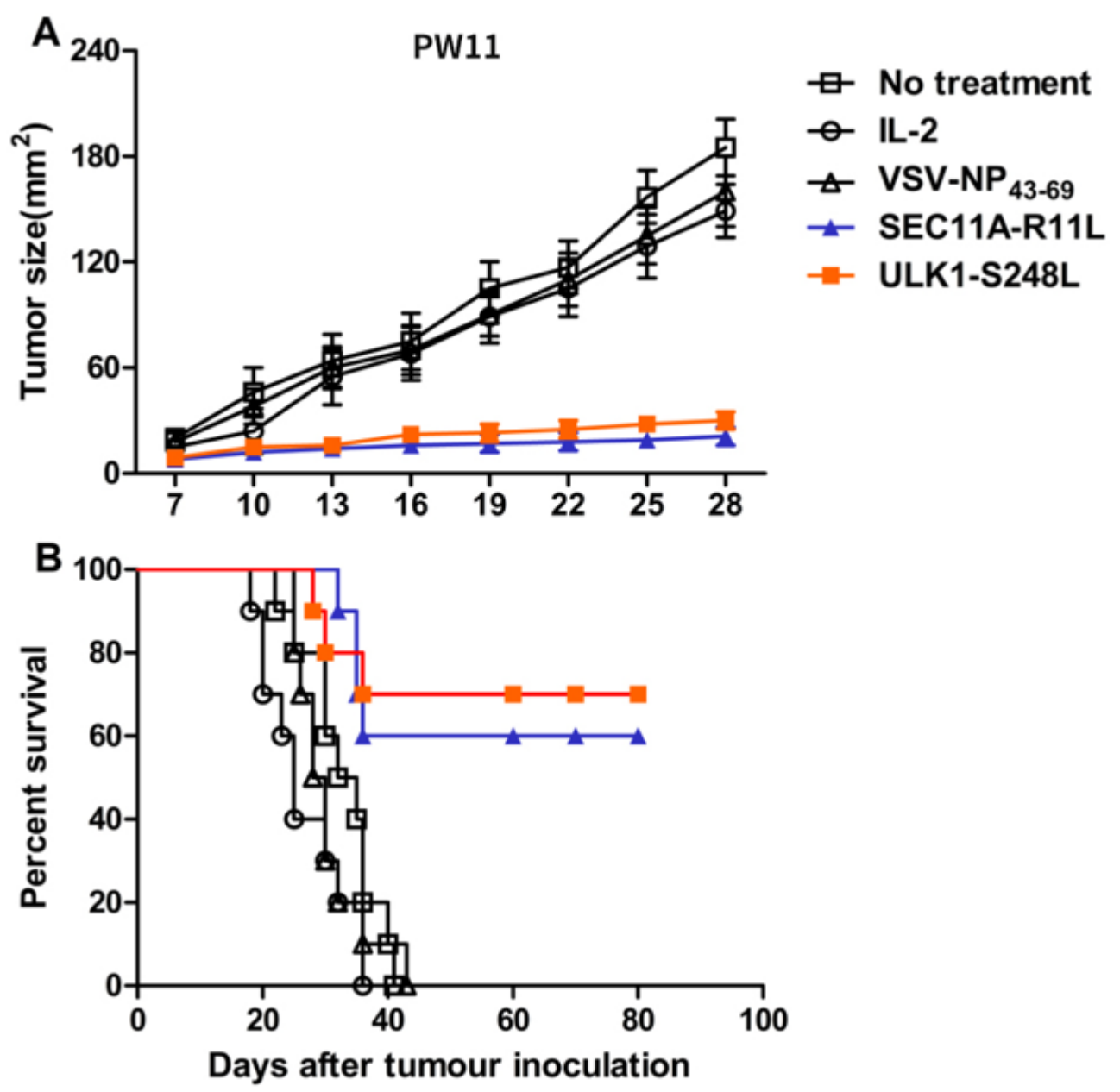

Figure 10

Adoptive immunotherapy of minigene-nucleofected SW480 tumor-bearing nude mice Small gene-nuclear transfected SW480 tumor cells ( $5 \times 106$ cells/mouse) were injected into the flanks of C57BL/6nu/nu mice, and three days later, HLA-matched immunization with SEC11AR11L and ULK1-S248L was performed. Splenocytes (1×108 cells/mouse) from HLA-A2.1/Kb-Tg mice were prepared as described in the Materials and Methods. The control group received only IL-2 or did not receive any treatment. (A) tumor growth was observed every 3 days and recorded as the mean tumor size (mm2). (B) Mouse survival after tumor inoculation was monitored ( $\mathrm{n}=10$ mice per group).

\section{Supplementary Files}

This is a list of supplementary files associated with this preprint. Click to download.

- Additionalfile3.pdf

- Additionalfile2.xls

- Additionalfile3.pdf

- Additionalfile1.pdf

- Additionalfile2.xls

- Additionalfile1.pdf 